\title{
Investigating students' strategies for interpreting quantum states in an upper-division quantum computing course
}

\author{
Josephine Meyer, ${ }^{1}$ Gina Passante, ${ }^{2}$ Steven Pollock, ${ }^{1}$ Michael Vignal,,${ }^{1}$ and Bethany Wilcox ${ }^{1}$ \\ ${ }^{1}$ Department of Physics, University of Colorado - Boulder, Boulder, CO 80309 \\ ${ }^{2}$ Department of Physics, California State University - Fullerton, Fullerton, CA 92831
}

\begin{abstract}
Significant focus in the PER community has been paid to student reasoning in undergraduate quantum mechanics. However, these same topics have remained largely unexplored in the context of emerging interdisciplinary quantum information science (QIS) courses. We conducted 15 exploratory think-aloud interviews with students in an upper-division quantum computing course at a large R1 university cross-listed in the physics and computer science departments. Focusing on responses to one particular problem, we identify two notably consistent problem-solving strategies across students in the context of a particular interview prompt, which we term Naive Measurement Probabilities (NMP) and Virtual Quantum Computer (VQC), respectively. Operating from a resources framework, we interpret these strategies as choices of coherent (and potentially mutually-generative) sets of resources to employ and available actions to perform.
\end{abstract}




\section{Introduction \& Motivation}

Quantum information science (QIS) coursework has, until recently, been restricted almost entirely to theoretical Ph.D.level coursework in physics. However, fueled by rapid growth in demand for jobs in quantum computing in the private sector as well as funding from the National Quantum Initiative Act of 2018, interdisciplinary QIS courses at the undergraduate or combined undergraduate/graduate level have begun to proliferate across US universities [1]. Such courses cover topics as wide-ranging as quantum algorithms, cryptography, quantum computer programming, laboratory work, and hardware design. Due to their relative novelty, little research has been done yet on such courses by the physics education research (PER) community. Recognizing this need, 32 scientists and professionals in QIS and adjacent fields signed an open letter calling for, among other provisions, the early involvement of education experts in curriculum development [2].

QIS education represents an intriguing but challenging frontier in PER for two reasons. First, QIS courses are often interdisciplinary across physics, computer science, or electrical engineering departments [3]. Interdisciplinary courses present a particular challenge to physics education research, since incoming students' backgrounds in linear algebra, algorithms, and quantum mechanics often vary wildly within and across courses. At the same time, the rapid proliferation of QIS courses presents an opportunity to influence instruction from the beginning. In this paper, we present the preliminary findings of an exploratory interview study designed to elucidate how students in QIS courses reason about the nature of quantum states in the context of quantum computing.

\section{Background and theoretical framework}

Attempts to extend PER frameworks developed in the context of traditional quantum mechanics courses to QIS date back to the mid-2000s. In 2003, Mermin [4] proposed a conceptual framework for teaching essential quantum mechanics to computer scientists in the context of quantum computing. In 2006, Singh [5] discussed specific student difficulties in quantum mechanics of particular relevance to quantum computing applications. Though explicit references to quantum information science are otherwise sparse in the literature, a number of subsequent publications have elucidated additional student difficulties relevant to QIS education [6-10]. Notably for our context, Passante et al. [6] found that more than half of surveyed undergraduate and beginning graduate students failed to distinguish between an ensemble of particles in a superposition state (in this case, $\frac{1}{\sqrt{2}}[|0\rangle+|1\rangle]$ ) and an ensemble of particles in a mixed state $(50 \%$ of particles randomly in the $|0\rangle$ state and $50 \%$ in the $|1\rangle$ state).

Prior work suggests that one common challenge for quantum mechanics students is the limited applicability of classical intuition to quantum mechanical situations [7, 11, 12]. Particular difficulties emerge in the contexts of superposition states $[6,10]$, where it is possible to conflate classical ran- domness (e.g. chaos) with the probabilistic nature of quantum mechanics [13]. Marshman and Singh [14] contextualize student difficulties in quantum mechanics by noting that students enter quantum mechanics courses with different backgrounds and levels of preparation, and that the process of learning quantum mechanics requires a Kuhnian [15] "paradigm shift" away from the 'realist' worldview of classical mechanics that often takes place in an incomplete or piecemeal fashion.

In interpreting QIS student reasoning, we have adopted a resources framework [16], founded on the ideas that resources must be acquired, activated, and then intentionally employed under particular circumstances for the purpose of problem-solving. One utility of the resources framework is that it appropriately reflects the diverse skills and experiences students bring to the interdisciplinary QIS classroom, and the sporadic and nonlinear process of undergoing such a paradigm shift. Additionally, the resources framework allows for the framing of naive approaches consistent with - though not necessarily indicative of - a realist worldview as reasonable and even expert-like depending on their use within the students' larger problem-solving strategy.

\section{Definition of "Strategy"}

For the purposes of this paper, we adopt the term "strategy" to describe phenomena emergent from our analysis of the interview data in the context of the resources framework. We conceptualize a strategy as a judgment, implicit or explicit, of (1) a coherent subset of resources to employ, and (2) an associated series or ensemble of steps or actions taken to string these resources together to accomplish a goal. Taken together, these two elements have the potential for a synergistic effect: the choice of resources to employ limits the actions that are possible and reasonable to take with those resources, while the strategy itself creates a context that primes a particular set of resources to be activated and available to employ. Our conception of "strategy" is primarily concerned with the coursegrained categories of resources activated (e.g. quantum gates or measurement as a whole) rather than fine-grained individual resources a student may or may not actually ever activate (such as the CNOT gate or the possibility of applying measurement only to a particular subset of qubits). Our analysis is likewise predicated on the observation that resource activation and usage is not random: a student's initial judgments (implicit or explicit) about what resources may be useful determine which resources are likely to be activated, and a student must also make a judgment about whether any given activated resource is productive to utilize.

Though we view the two elements listed above as characterizing a strategy, for the purposes of this paper we narrow our focus to strategies we observed with reasonable consistency and regularity across individuals in a similar context.

\section{Methodology}

We conducted think-aloud interviews of 15 students in an upper-division undergraduate quantum computing course at 
a large, predominantly-white R1 research university. Interviews were conducted in the last few weeks of the semester, after relevant material was covered. The course was crosslisted in the Physics and Computer Science departments, and the only formal prerequisite was completion of a linear algebra course. The course was co-taught by two instructors, one from Computer Science and one from Physics. Interview participants were paid respondents to an email request for research participants. Interviews were conducted over Zoom and transcribed using Otter.ai, with transcriptions verified manually as necessary. Given that QIS courses represent a largely new frontier for PER, our interview questions were necessarily exploratory, intended primarily to generate leads for future research. Preliminary analysis of our interview data suggested the existence of two (and possibly additional) reasoning patterns that - however arrived at - proceeded with surprising consistency from student to student and problem to problem once initiated.

Because this work is preliminary, this paper will focus specifically on the first think-aloud problem, closely inspired by a problem from a prior study [6] documenting student difficulties with superposition vs. mixed states. Students were asked to reason about the difference between two registers of qubits, with the prompt shown in Fig. 1: Register A (RA) in which the qubits were uniformly initialized into a superposition state and Register B (RB) in which they were randomly initialized into what experts would term a mixed state.

A correct response to the question in Fig. 1 is that RA and $\mathrm{RB}$ are not identical, and that they are distinguishable. From an information theory perspective, RA and RB are distinguishable by measuring the qubits in a basis other than the standard basis where the measurement probabilities of 0 and 1 are no longer 50/50 for RA. The most natural choice of basis is one commonly known as the Hadamard basis, of which the state of the qubits in RA is itself one of the basis states.

In practice, we observed that our interviewees and students in the class consistently used the term "measurement" to refer

\footnotetext{
Suppose a quantum computer has two identically-constructed registers of qubits, each containing a large number of qubits. In Register A, each of the qubits are in the state $\frac{1}{\sqrt{2}}[|0\rangle+|1\rangle]$. In Register $B$, the qubits have been randomly initialized so that approximately half of the qubits are in the state $|0\rangle$ and the other half are in the state $|1\rangle$.

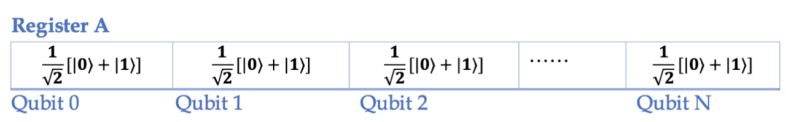

Register B (qubit states are randomly chosen)

\begin{tabular}{|l|l|l|l|l|}
\hline $50 \%$ probability $|0\rangle$ & $50 \%$ probability $|0\rangle$ & $50 \%$ probability $|0\rangle$ & $\ldots \ldots \ldots . .$. & $50 \%$ probability $|0\rangle$
\end{tabular} \begin{tabular}{llll|l|l|}
$50 \%$ probability $|1\rangle$ & $50 \%$ probability $|1\rangle$ & $50 \%$ probability $|1\rangle$ & & $50 \%$ probability $|1\rangle$ \\
\hline
\end{tabular} \begin{tabular}{llll}
\hline Qubit 0 & Qubit 1 & Qubit 2 & Qubit N
\end{tabular}

a) Are Registers A and B identical? (In other words, is the difference just semantics?) Why or why not?

b) Ok, Registers A and B are technically different. Is there any way we can distinguish them?

c) How might we go about trying to distinguish them with a quantum computer?
}

FIG. 1: Interview prompt provided to students. Students were offered the opportunity to use the Zoom whiteboard if requested. specifically to measurement in the standard basis $\{|0\rangle,|1\rangle\}$, though experts in QIS frequently describe measurements in arbitrary bases. Consistent with students' observed usage, for the purposes of this paper, we thus henceforth use the term "measurement" as shorthand for "measurement in the standard basis" unless otherwise noted.

Limiting ourselves to measurements in the standard basis as we observed students doing, the simplest equivalent protocol for distinguishing the registers in Fig. 1 is to apply the Hadamard gate ${ }^{1}$ - an elementary gate in QIS theory which is among the first quantum gates introduced - and then measure the qubits in the standard basis. If you apply this protocol, you will measure 0 for all qubits in RA and a 50/50 mix of 0 's and 1's in RB. Naturally, some students conceptualized more complex approaches, but all such approaches involved subjecting the qubits in each register to a particular sequence of quantum gates (or algorithms composed of gates) effecting a change of basis, followed by measurement.

As our first step in our analysis, we noted the existence of two distinct, coherent, and consistent patterns of student reasoning that were used by a large number of students despite diverse paths to arrive at the pattern. Over several rounds of qualitative coding, we conceptualized these two patterns as distinct strategies under the definition above. Throughout this process, we simultaneously refined our definitions of the term "strategy" itself and of the two specific strategies we outline to arrive at a coding schema for which instances of a given strategy could be unambiguously identified. Each strategy is defined in terms of characteristic steps and resources invoked (or not observed to be invoked). Because the full extent of student reasoning is not always articulated in a think-aloud interview, the specificity of our final definitions leaves any quantitative analysis vulnerable to undercount.

Interviewees ( $N=15)$ were asked to report their major(s) or intended major(s) and any prior physics courses taken. A slight majority (8) of students reported a major or intended major in physics or engineering physics, and 6 students reported a major or intended major in computer science. In addition, 3 students reported majoring in mathematics, and 3 students reported other engineering majors (students could report more one major). Also, 14 of the 15 students had completed at least one semester of college-level physics or equivalent, and 9 had completed a course in modern physics. Only 2 students had completed at least one semester of quantum mechanics, and 2 additional students were concurrently enrolled in their first semester of quantum mechanics.

\section{Results and Discussion}

In total, 11 of 15 students were able to arrive at a suitable protocol for distinguishing the Registers, with the interviewer providing reasonable guidance and prompting as he deemed appropriate. An additional 2 students were able to solve the problem upon review, after later problems in the interview had explicitly activated the resource of the Hadamard gate.

${ }^{1}$ The Hadamard gate, $H$, acts as a bidirectional map between the standard basis states $\{|0\rangle,|1\rangle\}$ and the Hadamard basis states $\left\{\frac{1}{\sqrt{2}}[|0\rangle \pm|1\rangle]\right\}$. 
For the remainder of this section, we articulate and describe the two strategies (Naive Measurement Probabilities, NMP, and Virtual Quantum Computer, VQC) that we observed students using consistently across this problem. We use qualitative analysis to establish the existence and widespread consistency of each strategy. We note that sole or final reliance on NMP is misleading in the context of this particular problem, but that NMP by itself was productive for answering other questions on the interview. Also, the strategies of NMP and VQC are not intended to be exhaustive; upon further analysis, we anticipate additional consistent strategies may emerge beyond the two discussed here.

\section{A. Strategy \#1: Naive Measurement Probabilities}

The first concrete strategy we present we term Naive ${ }^{2}$ Measurement Probabilities (NMP). This strategy is a straightforward first-order approach: the characteristic steps are to immediately square each of the coefficients in the quantum state to obtain the measurement probabilities in the basis provided (in practice, the standard basis) and to use these probabilities to help make physical sense of the state. The characteristic resource is directly applying (standard-basis) measurement to a given quantum state, in the absence of gates or algorithms. We make no inference from the use of NMP by itself whether the excluded resources are activated but strategically disregarded by the student, or simply not available to a student at the time. We code 8 of 15 students as invoking this strategy at some point during the duration of the problem. The following dialogue is characteristic of a student invoking NMP:

Interviewer: Can you tell me why [you think the two registers are the same]? Jacob: Because if you measure the plus state ${ }^{3}$, it's essentially just a 50 percent probability of 0 or 1. Interviewer: So, ok, so you're saying for any purpose, like, Registers $A$ and $B$ are just identical. We just, like, used different words to describe them here.

Jacob: Hmm, actually I think, um, no. If you measure Register A, then you get Register B, but before measuring they're different, I think.

Jacob's initial response above clearly demonstrates the elements of NMP: he reads off and squares the coefficients of the quantum state in RA and compares the resulting standardbasis measurement probabilities with those of RB. Jacob's use of measurement as a resource is explicit. His use of the resources of quantum gates or algorithms is conspicuously absent, on the other hand, given that the problem is framed in the context of qubits living inside a quantum computer. Note that by the last line, Jacob has clearly moved on from the strategy of NMP because he is invoking steps other than

\footnotetext{
${ }^{2}$ We use the term "naive" not as an indictment of NMP as a strategy, but only to acknowledge that NMP represents a relatively narrow conception of measurement probabilities compared to the basis-independent expert definition. ${ }^{3}|+\rangle \equiv \frac{1}{\sqrt{2}}[|0\rangle+|1\rangle]$
}

the characteristic steps of NMP, even though he is still solely invoking the resource of measurement.

\section{B. Strategy \#2: Virtual Quantum Computer}

We term the second observed strategy Virtual Quantum Computer (VQC). The characteristic resources of the VQC strategy are those physical resources available in a quantum computer that can be used to act on a qubit or qubits: quantum gates (or quantum algorithms that comprise compilations of gates). The standard-basis measurement operator is also available within a quantum computer and will often be invoked; however, we do not classify measurement as a characteristic resource for the purposes of defining VQC because the goal of the strategy might not be to output classical information. The characteristic actions of VQC are to apply various gates, sequences of gates, or algorithms to the state, chosen from the set of activated resources, and repeated as necessary or until the student moves on. The following dialogue is typical of a student invoking VQC:

Interviewer: ... Is there any way you could ... think of ... experimentally distinguishing the registers? Morgan: So just to give you my thought process here, I'm thinking about gates that I can apply. Um, oh, actually. [Expletive], you can only measure once - sorry, I shouldn't have sworn. So my initial thought was like, if you apply a Hadamard to Register A, you're going to get 0 for every single one of these, right?

Notice that when Morgan was asked if there was a way to distinguish RA and RB, they invoked VQC without hesitation. Morgan explicates their thought process in terms of trying various gates, and their aside also shows that they are conceptualizing measurement as a potentially employable resource. In the dialogue that follows, upon recognizing that the Hadamard gate will rotate all qubits in RA into the $|0\rangle$ state, Morgan tests the Hadamard gate on the qubits in RB. Noting that the resulting states are clearly distinguishable upon measurement (given large enough $N$ ), they have successfully identified a working protocol for distinguishing the registers.

Other examples of VQC will be discussed in aspects of Tom and Ella's dialogues below. In total, we identified 14 of 15 students as invoking the VQC strategy. Jacob is the only example of a student who did not invoke VQC for this problem, despite heavy prompting to think about gates; he was one of only two students not to complete the problem.

\section{Strategies and Resource Activation}

Though a strategy as we have defined it is distinct from the set of resources it employs, the use of a strategy requires students to have actually activated enough of the resources for the steps of the strategy to make sense. Thus, the use of a strategy may be a spontaneous decision based on resources already activated, or its use may come as a reaction to an external prompt that activates the necessary resource(s). We ex- 
amine how this can occur through two contrasting case studies, both involving students quickly transitioning into VQC upon apparent activation of gates as an available resource:

Tom: ...If we were just to measure each-no, because we can only measure 0 or 1 . But if we did-(slowly) if we were to apply, um, say an N-fold Hadamard to each and then measure, the first register would give us all 0's. The second one would be still 50/50.

Here, Tom's first sentence suggests he is initially trying to apply just the resource of measurement, though his dia$\log$ is too fast and sparse to establish NMP. However, he then takes a slight pause to think. After some time, but without the need for external prompting, he activates the resource of the Hadamard gate. Once he activates the Hadamard gate as a resource that can be applied to the state and chooses to employ it, the remaining necessary moves - applying the Hadamard gate to the qubits in each register, performing a measurement on the resulting states, and noting that the measurement will output different results for each measurement - immediately fall into place.

Ella, below, incorrectly answers (with some hesitation in her voice) that RA and RB are indistinguishable in an instance of NMP. As soon as the interviewer prompts her to consider the quantum state in the context of a quantum computer - presumably either activating the appropriate resources or prompting her to employ them - Ella transitions to VQC, interrupts the interviewer midsentence, and immediately solves the problem.

Interviewer: So yeah, [Registers $A$ and $B$ are] technically different. Is there any way we can actually distinguish them? Ella: Hmm, um, no - not really. Interviewer: Yeah, why not? Ella: Um, because if we do measure them, then we would get the same behavior? Interviewer: Sure. Cool, I agree with that statement, that if you measure them you get the same behavior. (Pauses) Let's say, so these are in a quantum computer. Um - Ella: Mmmm (nods) Interviewer: - is there any quantum computational protocol we could use to try to - Ella: Uh, I guess if we applied Hadamard gates to, um, Register $A$, then they would all become, they would become $|0\rangle$ state. And then for $B$, they would still, have, um, you would measure 0 and 1 equally still.

Ella and Tom's examples show that the VQC strategy can be either spontaneously chosen or prompted by an external stimulus. Regardless, once both Ella and Tom have begun using the strategy - however they got there - the remaining steps fall naturally into place. Not all students, of course, immediately stumbled on a viable strategy on their first try applying various gates or algorithms, but their process nevertheless proceeded along similar lines.

\section{Conclusions}

We have identified two strategies, which we term Naive Measurement Probabilities (NMP) and Virtual Quantum Computer (VQC), we observed employed with consistency across a sizable fraction of student interviews. For our purposes, we defined a strategy as a well-defined series of steps employing (or intentionally not employing) a coherent set of resources - as a phenomenological instrument for interpreting patterns of student reasoning that appear surprisingly coherent and consistent across students. Our conception of a strategy is specifically developed in the context of two such examples employed throughout the solution of a particular problem. Extending this framework beyond the context of the examples from which it was developed is beyond the scope of our current analysis, though we suspect we will find other coherent strategies under this definition emergent from our analysis of the rest of the interview problems.

At minimum, the documentation of these two strategies is itself useful to the educator. An instructor, for instance, may elect to show explicit examples of precisely when NMP is productive and when overreliance on NMP can fail, so that students' practical success in employing NMP in many contexts does not inspire false confidence.

At this time, since this preliminary investigation was limited to students of a single course, we make no claims as to whether the specific observed strategies apply universally across courses. In particular, students' restriction of measurement to the standard basis, as we observed in NMP, may be an artifact of this particular course. However, because gates and measurement are foundational resources in QIS - and because there is no evidence that students were explicitly taught to use either of these strategies - there is reason to think that strategies similar to the ones we documented may nevertheless be found among students in other courses, and we intend to explore this possibility in follow-up research.

Our findings suggest additional avenues for further work. With more students and more focused interviews, for instance, we hope to meaningfully compare the strategies physics and computer science students employ within the same quantum computing class to identify potential differences in resource activation. Building on our preliminary analysis, we also intend to use follow-up interviews to probe more fine-grained resource activation and utilization. Finally, our preliminary analysis given here is necessarily phenomenological as it is among the first inquiries in PER into QIS student reasoning. In future research, we intend to conduct more detailed interview studies in an effort to better connect our findings in this paper with more specific established theoretical frameworks in PER under the umbrella of the resources framework. Though we do not yet have enough data to take a definitive stance, one possibility is that the strategies we identify, particularly VQC, might represent epistemic games [17] at least under the framework of Redish [18].

\section{Acknowledgments}

Special thanks to Giaco Corsiglia for his work coordinating joint interviews, and for the PER@C members who offered valuable feedback. This research is funded by the CU Dept. of Physics, the NSF Graduate Research Fellowship Program, and NSF Grants No.’s 2012147 and 2011958. 
[1] M. Fox, B. Zwickl, and H. Lewandowski. Preparing for the quantum revolution: What is the role of higher education? Phys. Rev. Phys. Educ. Res. 16, 020131 (2020).

[2] C. Aiello et al. Achieving a quantum smart workforce. Quantum Sci. Technol. 6, 030501 (2021).

[3] B. Cervantes et al. An overview of quantum information science courses at US institutions. Submitted to Proceedings of the Physics Education Research Conference (2021).

[4] N. Mermin. From Cbits to Qbits: Teaching computer scientists quantum mechanics. Am. J. Phys. 71, 23 (2003).

[5] C. Singh. Helping students learn quantum mechanics for quantum computing. In Proceedings of the Physics Education Research Conference (2006).

[6] G. Passante, P. Emigh, and P. Shaffer. Student ability to differentiate between superposition states and mixed states in quantum mechanics. Phys. Rev. ST Phys. Educ. Res. 11, 020135 (2015).

[7] C. Singh and E. Marshman. Review of student difficulties in upper-level quantum mechanics. Phys. Rev. ST Phys. Educ. Res. 11, 020117 (2015).

[8] G. Zhu and C. Singh. Surveying students' understanding of quantum mechanics in one spatial dimension. Am. J. Phys. 80, 252 (2012).

[9] A. Kohnle and E. Deffebach. Investigating student understanding of quantum entanglement. In Proceedings of the Physics Education Research Conference (2015).

[10] T. Wan, P. Emigh, and P. Shaffer. Probing student reasoning in relating relative phase and quantum phenomena. Phys. Rev.
Phys. Educ. Res. 15, 020139 (2019).

[11] C. Singh and G. Zhu. Cognitive issues in learning advanced physics: An example from quantum mechanics. In The Proceedings of the Physics Education Research Conference (2009).

[12] B. Dreyfus, E. Sohr, A. Gupta, and A. Elby. "Classical-ish": Negotiating the boundary between classical and quantum particles. In The Proceedings of the Physics Education Research Conference (2015).

[13] C. Baily and N. Finkelstein. Development of quantum perspectives in modern physics. Phys. Rev. ST Phys. Educ. Res. 5, 010106 (2009).

[14] E. Marshman and C. Singh. Framework for understanding the patterns of student difficulties in quantum mechanics. Phys. Rev. ST Phys. Educ. Res. 11, 020119 (2015).

[15] T. Kuhn. The structure of scientific revolutions. Chicago: University of Chicago Press (1962).

[16] D. Hammer, Student resources for learning introductory physics, Am. J. Phys. 68, (2000).

[17] A. Collins and W. Ferguson. Epistemic forms and epistemic games: Structures and strategies to guide inquiry. Educational Psychologist 28, 1 (1993).

[18] E. Redish. A theoretical framework for physics education research: Modeling student thinking. In The Proceedings of the Enrico Fermi Summer School in Physics, Course CLVI, Italian Physical Society (2004). 